\title{
Canny based DRLSE Algorithm for Segmentation
}

\author{
Arati J. Vyavahare, Ph.D \\ Professor, E\&TC Department, \\ PES's Modern College of Engineering, \\ Pune-05, India
}

\begin{abstract}
Image segmentation is typically used to locate objects and boundaries in images. The objective of this application is the segmentation of different medical images. Edge detection significantly reduces the amount of data and filters out useless information, while preserving the important structural properties in an image. Medical image segmentation is one of the most important parts of clinical diagnostic tools. However, the process of accurate segmentation of these images is very important and crucial for a correct diagnosis by clinical tools.

In this paper canny operator based Distance Regularization Level Set Evolution Algorithm (DRLSE) is used. The distance regularization effect eliminates the need for reinitialization and thereby avoids its induced numerical errors. First canny operator is used to determine the edges and edge directions. Then a new variation level set formulation is used in which the regularity of the level set function is intrinsically maintained during the level set evolution. The level set evolution is derived as the gradient flow that minimizes energy functional with a distance regularization term and an external energy that drives the motion of the zero level set toward desired locations. The distance regularization term is defined with a potential function such that the derived level set evolution has a unique forward-and-backward (FAB) diffusion effect, which is able to maintain a desired shape of the level set function, particularly a signed distance profile near the zero level set. Proposed algorithm is used for database of different medical images such as Brain, Eye \& Comparison of the same algorithm for those images by using different parameters is done.
\end{abstract}

\section{Keywords}

Canny operator, Distance Regularization Level Set Evolution Algorithm (DRLSE)

\section{INTRODUCTION}

The goal of segmentation is to simplify and/or change the representation of an image into something that is more meaningful and easier to analyze. Image segmentation is typically used to locate objects and boundaries (lines, curves, etc.) in images [3]. Edge detection is a very important area in the field of computer vision [10][11][12]. Edge detection significantly reduces the amount of data and filters out useless information, while preserving the important structural properties in an image. Edge detection refers to the process of identifying and locating sharp discontinuities in an image. The effect of edge detection has a great influence on image segmentation. The canny edge detector has a simple approximate implementation in which edges are marked at maxima in gradient magnitude of a Gaussian-smoothed image [4]. The performance of the canny algorithm depends heavily on the adjustable parameter ' $\sigma$ ' which is the standard deviation for the gaussian filter, and the threshold values, 'T1' and ' $\mathrm{T} 2$ '. Canny operator overcomes the disadvantages of tradition edge detection operators, such as roberts operator, sobel operator, prewitt operator. It can detect almost all edges, and is one of the best edge detection algorithms. So, considering the different medical images database, a canny operator based distance regularization level set evolution (DRLSE) algorithm. The algorithm combines the advantages of canny operator which can orient the boundary accurately and the idea that DRLSE algorithm continuously evolves the boundary in image space. Proposed algorithm is used for database of different medical images such as Brain, Blood cell, Eye \& Comparison of the same algorithm for those images by using different parameters is done [1].

\section{METHODOLOGY}

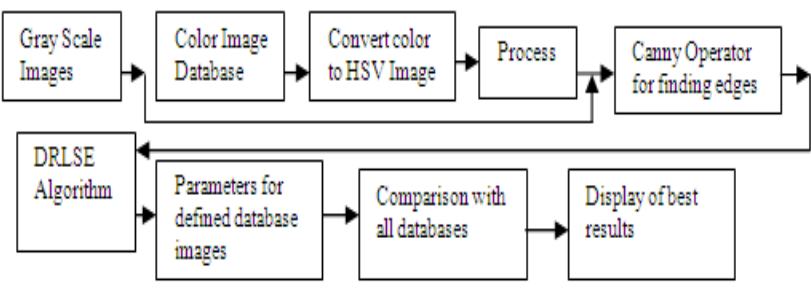

Fig 1. Block Diagram of Canny Operator Based DRLSE Algorithm

\subsection{Canny Edge Detection Algorithm}

The canny edge detection operator was developed by John F.Canny in 1986 and uses a multi-stage algorithm to detect a wide range of edges in images. Most importantly, canny also produced a computational theory of edge detection explaining why the technique works. canny's aim was to discover the optimal edge detection algorithm [7].

The algorithm runs in 5 separate steps:

1. Smoothing: Blurring of the image to remove noise.

2. Finding gradients: The edges should be marked where the_gradients of the image has large magnitudes.

3. Non-maximum suppression: Only local maxima should be_marked as edges.

4. Double thresholding: Potential edges are determined by_thresholding.

5. Edge tracking by hysteresis: Final edges are determined by_suppressing all edges that are not connected to a very certain strong) edge.

\subsection{DRLSE}

In level set methods, a contour (or more generally a hypersurface) of interest is embedded as the zero level set of an LSF. Although the final result of a level set method is the zero level set of the LSF, it is necessary to maintain the LSF in a good condition, so that the level set evolution is stable 
and the numerical computation is accurate. This requires that the LSF is smooth and not too steep or too flat (at least in a vicinity of its zero level set) during the level set evolution. This condition is well satisfied by signed distance functions for their unique property $|\nabla \varnothing|=1$, which is referred to as the signed distance property. For the 2-D case as an example, we consider a signed distance function $\mathrm{z}=\varnothing(\mathrm{x}, \mathrm{y})$ as a surface. Then, its tangent plane makes an equal angle of 45 with both the -plane and the -axis, which can be easily verified by the signed distance property $|\nabla \varnothing|=1$. For this desirable property, signed distance functions have been widely used as level set functions in level set methods. In conventional level set formulations, the LSF is typically initialized and periodically reinitialized as a signed distance function. In this section, we propose a level set formulation that has an intrinsic mechanism of maintaining this desirable property of the LSF [8][9].

\subsection{DRLSE Mechanism}

2.3.1 Energy Formulation with Distance Regularization Let $\emptyset: \Omega \rightarrow \mathcal{R}$ be a LSF defined on a domain. We define energy functional $\varepsilon(\varnothing)$ by

$$
\varepsilon(\varnothing)=\mu \mathcal{R}_{\mathrm{p}}(\varnothing)+\varepsilon_{\text {ext }}(\varnothing)
$$

where $\mathcal{R}_{\mathrm{p}}(\varnothing)$ is the level set regularization term defined in the following, $\mu>0$ is a constant, and $\varepsilon_{\text {ext }}(\varnothing)$ is the external energy that depends upon the data of interest. The level set regularization term $\mathcal{R}_{\mathrm{p}}(\varnothing)$ is defined by

$$
\mathfrak{R}_{p}(\phi) \triangleq \int_{\Omega} p\left(\nabla_{\phi}\right) d x
$$

Where $p$ is a potential (or energy density) function. $\mathrm{p}:[0, \infty) \rightarrow \mathcal{R}$ The $\varepsilon_{\text {ext }}(\varnothing)$ energy is designed such that it achieves a minimum when the zero level set of the LSF $\varnothing$ is located at desired position.

The level set regularization term $\mathcal{R}_{\mathrm{p}}(\varnothing)$ is referred to as a distance regularization term. $\mathcal{R}_{\mathrm{p}}(\varnothing)$ is used for maintaining the signed distance property of the LSF. A simple and straightforward definition of the potential $p$ for distance regularization is

$$
p=p_{1}(s) \triangleq \frac{1}{2}(s-1)^{2}
$$

For satisfying the condition of the LSF, the potential function must have minimum points $s=1 \& \quad s=0$.Such a potential is a double-well potential as it has two minimum points (wells). Double-well potential offers some appealing theoretical and numerical properties of the level set evolution.

\subsubsection{Gradient Flow for Energy Minimization}

A standard method to minimize energy functional $F(\varnothing)$ is to find the steady state solution of the gradient flow equation

$$
\frac{\partial \phi}{\partial t}=-\frac{\partial F}{\partial \phi}
$$

Where $\frac{\partial F}{\partial \varnothing}$ is the Gateaux derivative of the functional $F(\varnothing)$. The evolution of the time-dependent function $\varnothing(\mathrm{x}, \mathrm{t})$ is in the opposite direction of the Gâteaux derivative, i.e., which is the steepest descent direction of the functional. Therefore, the gradient flow is also called steepest descent flow or gradient descent flow.
The distance regularization effect in DRLSE can be seen from the gradient flow of the energy $\mu \mathcal{R}_{\mathrm{p}}(\varnothing)$.

This flow can be expressed in standard form of a diffusion. This diffusion is not a usual diffusion because the diffusion rate can be positive or negative for the potential used in DRLSE.

When $\mathrm{d}_{\mathrm{p}}(|\nabla \emptyset|)$ is positive, the diffusion is forward diffusion, which decreases $|\nabla \emptyset|$. When $\mathrm{d}_{\mathrm{p}}(|\nabla \emptyset|)$ is negative, the diffusion is backward diffusion, which increases $|\nabla \emptyset|$. Such diffusion is called a Forward-and-Backward (FAB) diffusion.

The sign of the function indicates the property of the FAB diffusion term in the following two cases:

Case1:- For, $|\nabla \emptyset|>1$,the diffusion rate is positive, and the diffusion (11) is forward, which decreases $|\nabla \emptyset|$;

Case2:- For $|\nabla \emptyset|<1$, the diffusion rate is negative, and the diffusion becomes backward, which increases $|\nabla \emptyset|$.

2.3.3 Double-Well Potential for Distance Regularization There is a specific construction of the double-well potential p2 as

$\mathrm{p}_{2}(\mathrm{~s})=\left\{\begin{array}{ll}\frac{1}{(2 \pi)^{2}}(1-\cos (2 \pi \mathrm{s})), & \text { if } \mathrm{s} \leq 1 \\ \frac{1}{2}(\mathrm{~s}-1)^{2}, & \text { if } \mathrm{s} \geq 1\end{array} \ldots \ldots\right.$.

This potential $p_{2}(s)$ has two minimum points at $\mathrm{s}=0$ and $\mathrm{s}=1$ It is easy to verify that is twice differentiable in , with the first and second derivatives given by

$$
\mathrm{p}^{\prime}{ }_{2}(\mathrm{~s})= \begin{cases}\frac{1}{2 \pi} \sin (2 \pi \mathrm{s}), & \text { if } \mathrm{s} \leq 1 \\ \mathrm{~s}-1, & \text { if } \mathrm{s} \geq 1\end{cases}
$$

and

$\mathrm{p}^{\prime \prime}{ }_{2}(\mathrm{~s})= \begin{cases}\cos (2 \pi \mathrm{s}), & \text { if } \mathrm{s} \leq 1 \\ 1, & \text { if } \mathrm{s} \geq 1\end{cases}$

which verifies the boundedness of the diffusion rate for the potential $\mathrm{p}=\mathrm{p}_{2}$, indicates the property of the $\mathrm{FAB}$ diffusion term in the following three cases:

Case1:- For $|\nabla \emptyset|>1$ the diffusion rate is positive, and the diffusion (11) is forward, which decreases $|\nabla \emptyset|$

Case2:- For, $\left(\frac{1}{2}\right)<|\nabla \emptyset|<1$ the diffusion rate is negative, and the diffusion becomes backward, which increases $|\nabla \emptyset|$;

Case3:- For , $|\nabla \emptyset|<(1 / 2)$ the diffusion rate is positive, and the diffusion is forward, which further decrease down to zero. The key differences between the FAB diffusions with potentials and are the boundedness of the corresponding diffusion rate and the diffusion behavior for the case , as can be seen from the previous descriptions for both cases.

\subsubsection{Distance Regularization Effect}

From the distance regularization effect of DRLSE by simulating the FAB diffusion (11) with the initial function being a binary step function. The binary step function is defined by

$$
\emptyset_{0}(x)=\left\{\begin{array}{cc}
-c_{0}, & \text { if } x \in R_{0} \\
c_{0}, & \text { otherwise }
\end{array}\right.
$$

where $c_{0}$ is a constant, and $R_{0}$ is a region in the domain.

Even with the irregularity of the binary step function, the FAB [21] diffusion (11) is able to evolve the LSF into a function with desired regularity. It is worth noting that a binary step function can be generated extremely efficiently. 


\subsection{Edge-Based Active Contour Model in Distance Regularized Level Set}

\section{Formulation}

Let $I$ be an image on a domain, we define an edge indicator function by

$g \triangleq \frac{1}{1+\left|\nabla G_{\sigma} * I\right|^{2}}$

where $G_{\sigma}$ is a Gaussian kernel with a standard deviation. The convolution is used to smooth the image to reduce the noise. This function usually takes smaller values at object boundaries than at other locations. For Let $\emptyset: \Omega \rightarrow \mathrm{R}$, we define an energy functional by

$\varepsilon(\varnothing)=\mu \mathcal{R}_{\mathrm{p}}(\varnothing)+\lambda \mathcal{L}_{\mathrm{g}}(\varnothing)+\alpha \mathcal{A}_{\mathrm{g}}(\varnothing)$

Where $\lambda>0$ and $\alpha \in \mathrm{R}$ are the coefficients of the energy functionals and, which are defined by

$$
\mathcal{L}_{\mathrm{g}}(\emptyset) \triangleq \int_{\Omega} g \delta(\phi)|\nabla \phi| d x \quad \ldots \ldots \ldots \ldots
$$

$\mathcal{A}_{\mathrm{g}}(\varnothing) \triangleq \int_{\Omega} g H(-\phi) d x$

where $\delta$ and $\mathrm{H}$ are the Dirac delta function and the Heaviside function respectively. [10]

\subsection{Algorithm of Canny operator based}

\section{DRLSE algorithm [2]:-}

Step1:-Used Database of medical images such as Brain, eye as input.

Step2:- For edge detection used different operators. For better accuracy detected edges should be more and close to real edge. So, Compare different operators Prewitt, Sobel, Roberts $\&$ Canny Operators for finding edges.

Step3:-If image is grayscale Find edges by Canny operator.

Step4:-If image is colored then convert it into HSV color space.

Step5:- Then find edges by Canny operator.

Step6:- Then find seed point for zero level contour.

Step7:- DRLSE in which the regularity of the level set function is intrinsically maintained during the level set evolution. Then start level set evolution by using DRLSE means distance regularized level set Evolution.

Step6:- Then above steps tested on database of Brain tumor, blood cell, eye images.

Step7:- Compare different types of images by using different parameters

\section{EXPERIMENTAL RESULTS}

This section shows the results of the DRLSE model (30) for both synthetic and real images. There are parameters, and in this model, and the time step for the implementation. The model is not sensitive to the choice of and, which can be fixed for most of applications. Unless otherwise specified, these parameters are fixed as, and in this paper. The parameter needs to be tuned for different images. A nonzero gives additional external force to drive the motion of the contour, but the resulting final contour may slightly deviate from the true object boundary due to the shrinking or expandi ng effect of the weighted area term. To avoid such deviation, one can refine the final contour by further evolving the contour for a few iterations with the parameter. For images with weak object boundaries, a large value of may cause boundary leakage, i.e., the active contour may easily pass through the object boundary. Therefore, for images with weak object boundaries, the value of should be chosen relatively small to avoid boundary leakage.

\subsection{Comparison between Canny Operators with Prewitt, Sobel, Roberts by using color image.}

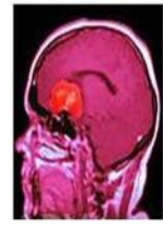

(a)

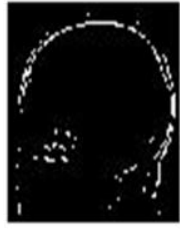

(e)

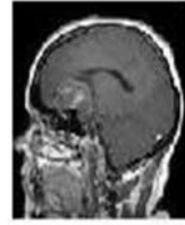

(b)

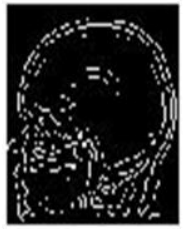

(f)

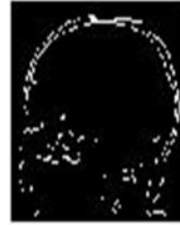

(c)

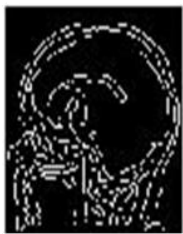

(g)

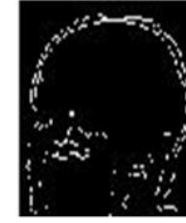

(d)
Fig 2 (a)Original Image (b)Grayscale Image (c)Prewitt (d)Sobel (e)Roberts (f)LOG (g)Canny

\subsection{Final algorithm checked for database of eye images.}

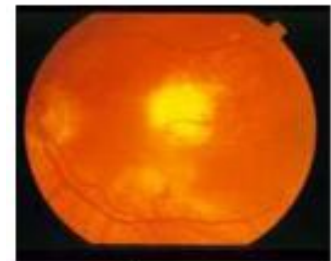

(a)

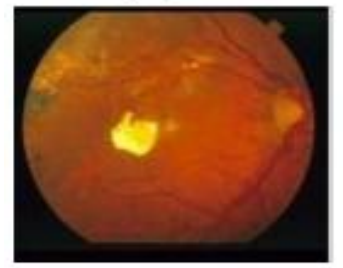

(b)

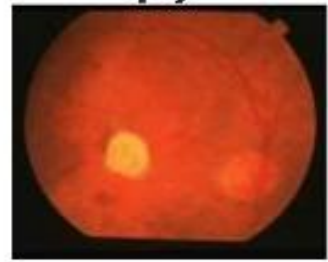

(c)

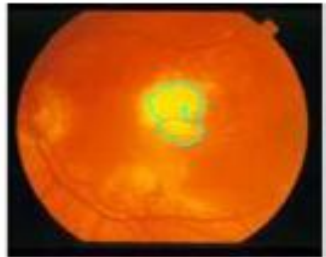

(f)

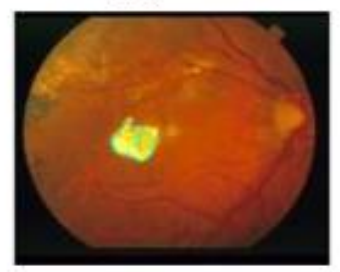

(g)

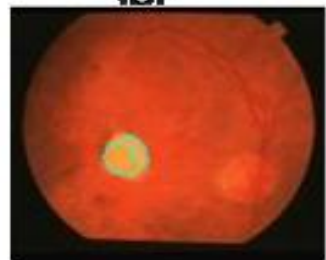

(h)
Fig. 3 (a),(b),(c) Original color images \& (f),(g),(h) Segmented image 


\subsection{Final algorithm checked for database of brain tumor images.}

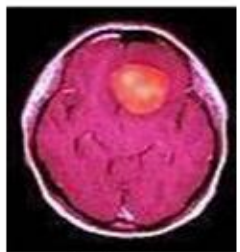

(a)

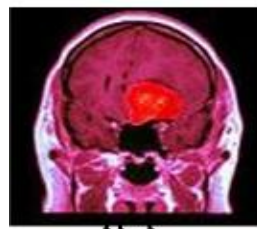

(b)

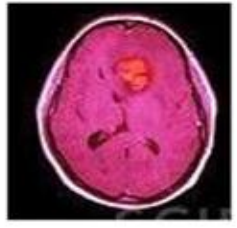

(c)

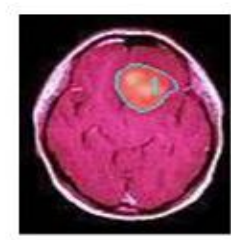

(f)

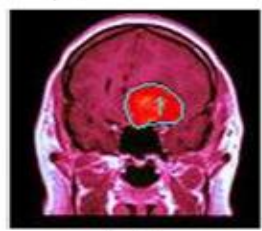

(g)

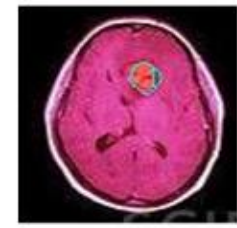

(h)
Fig. 4 (a),(b),(c) Original Color Images \& (f),(g),(h) Segmented Images

Table 1. Comparison between original \& raw images of eye with parameters.

\begin{tabular}{|c|c|c|c|c|c|}
\hline 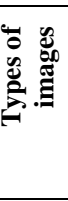 & $\begin{array}{l}\text { Para } \\
\text {-meters }\end{array}$ & E1 & E2 & E3 & E4 \\
\hline \multirow{8}{*}{ 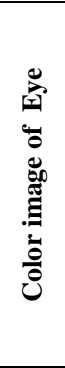 } & Idx & 1 & 2 & 1 & 1 \\
\hline & Area & 472 & 244 & 259 & 597 \\
\hline & Centroid & 6776 & $\begin{array}{l}112 \\
72\end{array}$ & $\begin{array}{l}56.5 \\
67.5\end{array}$ & $\begin{array}{l}81.5 \\
57.5\end{array}$ \\
\hline & Perimeter & 72 & 56 & 51 & 85 \\
\hline & Solidity & 0.786 & 0.7625 & 0.801 & 0.7703 \\
\hline & $\begin{array}{l}\text { Compactnes } \\
\mathrm{s}\end{array}$ & 10.98 & 12.852 & $\begin{array}{l}10.04 \\
2\end{array}$ & 12.102 \\
\hline & Form factor & 5.931 & 3.0662 & 3.254 & 7.502 \\
\hline & $\begin{array}{l}\text { Elapsed } \\
\text { time }\end{array}$ & ---- & 4.018 & 4.575 & 6.038 \\
\hline \multirow{8}{*}{ 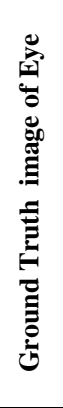 } & Idx & 1 & 2 & 1 & 1 \\
\hline & Area & 552 & 325 & 330 & 713 \\
\hline & Centroid & $\begin{array}{l}65.5 \\
77.5\end{array}$ & $\begin{array}{l}116 \\
68\end{array}$ & $\begin{array}{l}58.5 \\
65.5\end{array}$ & 8053.5 \\
\hline & Perimeter & 83 & 61 & 63 & 87 \\
\hline & Solidity & 0.761 & 0.923 & 0.748 & 0.788 \\
\hline & $\begin{array}{l}\text { Compactnes } \\
\mathrm{s}\end{array}$ & 12.48 & 11.449 & 12.07 & 10.615 \\
\hline & Form factor & 6.936 & 4.084 & 4.146 & 8.959 \\
\hline & $\begin{array}{l}\text { Elapsed } \\
\text { time }\end{array}$ & ---- & 0.197 & 0.178 & 0.173 \\
\hline
\end{tabular}

Table 2. Comparison between original \& raw images of brain tumor with parameters.

\begin{tabular}{|c|c|c|c|c|}
\hline 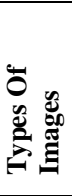 & $\begin{array}{l}\text { Param- } \\
\text { Eters }\end{array}$ & B1 & B2 & B3 \\
\hline \multirow{8}{*}{ 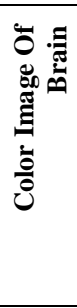 } & Idx & 1 & 1 & 1 \\
\hline & Area & 429 & 354 & 164 \\
\hline & Centroid & $\begin{array}{l}53.5 \\
39.0\end{array}$ & $\begin{array}{l}55.0 \\
39.5\end{array}$ & $\begin{array}{l}59.5 \\
37.5\end{array}$ \\
\hline & Perimeter & 63 & 59 & 38 \\
\hline & Solidity & 0.72 & 0.77 & 0.84 \\
\hline & Compactness & 9.25 & 9.83 & 8.80 \\
\hline & Form Factor & 5.39 & 4.45 & 2.06 \\
\hline & Elapsed Time & 5.34 & 7.59 & 5.45 \\
\hline \multirow{10}{*}{ 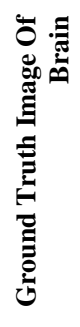 } & & & & \\
\hline & Idx & 1 & 1 & 1 \\
\hline & Area & 424 & 359 & 160 \\
\hline & Centroid & 51.5 & 54.5 & 57.5 \\
\hline & & 39.5 & 39.5 & 37.5 \\
\hline & Perimeter & 68 & 60 & 43 \\
\hline & Solidity & 0.88 & 0.90 & 0.82 \\
\hline & Compactness & 10.91 & 10.03 & 11.56 \\
\hline & Form Factor & 5.33 & 4.51 & 2.01 \\
\hline & Elapsed Time & 0.18 & 0.18 & 0.17 \\
\hline
\end{tabular}

\section{CONCLUSION}

From the comparison of operators results shows that, the Canny's edge detection algorithm performs better than all these operators under almost all scenarios.

The canny operator based DRLSE algorithm is tested for Brain, Eye images. For finding accuracy of algorithm Quality Metrics are used. Different parameters are calculated for original \& ground truth images. Error is calculated from those parameters. For proper segmentation calculated error should be zero or near to zero.

In the experiment canny operator based DRLSE algorithm is tested for number of images of Brain tumor. Out of those images, three are near to 0 error for maximum number of parameters.

For diabetic retinopathy same algorithm is tested for number of images. Out of which, segmented output there is some incorrect exudates detection which is due to artifacts, which appear similar to exudates.

Therefore, from above result we can say that algorithm gives meaningful, reasonable and effective segmentation output for brain images.

\section{REFERENCES}

[1] Chunming Li, Chenyang Xu, "Distance Regularized Level Set Evolution and Its Application to Image Segmentation"IEEE Trans. on Image Processing,vol.19,No.12,December 2010

[2] Xujja Qin,Jionghui jiang, Weihong wang "Canny Operator based level set segmentation algorithm for medical images," IEEE, ,2007, pp.892-895.

[3] Rajeshwar Dass, Priyanka, Swapna Devi "Image Segmentation Techniques" IJECT ISSN : 2230-7109 ISSN : 2230-9543 Vol. 3, Issue 1, March 2012

[4] Saket Bhardwaj, Ajay Mittal "A Survey on Various Edge Detector Techniques" Procedia Technology 4, 2012, 220 $-226$ 
[5] ZD. Xue, LJ. Li, ZY. Li, "Segment Virtual Human Slice Data Using SVM". Application Research of Computers, 14(4), 2006,pp.45-47 (In Chinese).

[6] S. Osher and J. Sethian, "Fronts Propagating with Curvature Dependent Speed: Algorithms Based On the Hamilton-Jacobi Formulation". Journal of Computation physics, 79(1),1998,pp.12-49.

[7] J.Canny, "A Computational Approach to Edge Detection". IEEE Transactions on Pattern Analysis and Machine Intelligence, 8(6),1986, pp.679-698.

[8] M. Sussman, P. Smereka, S. Osher, "A level set approach for computing solutions to incompressible two-phase flow". Journal of Computation physics, 144(1), 1994 , pp.146-149.
[9] J. Sethian, A Theory, algorithms and applications of Level Set methods for propagating interface. Cambridge University Press, Cambridge UK, 1995.

[10] Punam Thakare "A Study of Image Segmentation and Edge Detection Techniques", International Journal on Computer Science and Engineering, Vol 3, No.2, 899904, (2011.

[11] V. Torre and T. A. Poggio. "On edge detection". IEEE Trans. Pattern Anal. Machine Intell., vol. PAMI-8, no.2, Mar. 1986, pp. 187-163.

[12]T.A. Mohmoud; S.Marshal, "Edge -Detected Guided Morphological Filter for Image sharpening, Hindawi Publishing orporation EURASIP Journal on image and video Processing volume 2008 